\title{
HIV Seroprevalence Among Surgical Specialty Outpatients at a Teaching Hospital
}

\author{
*Omole-Ohonsi A FWACS \\ **Nwokede E E FMCPATH
}

*Department of Obstetrics and Gynaecology, Bayero University/Aminu Kano Teaching Hospital, Kano, Nigeria.**Department of Micobiology, Benue State University, Makurdi, Nigeria.

Correspondence to: Dr. Omole-Ohonsi A,

P. O. Box 14578, General Post Office,

Kano, Nigeria.Email: aomohonsi@yahoo.com. Tel: +234 -080-37870540

\begin{abstract}
Background: HIV infection rate is higher in densely populated and heavily industrialized communities like Kano. The risk of transmission of the infection from patients to health workers, and from mother to child is very high among antenatal women and surgical patients if their HIV status is not known, because of the higher probability of breaching the universal precautions.
\end{abstract}

Objective: To determine the sero-prevalence of Human Immunodeficiency Virus (HIV) infection among Surgical Specialty outpatients Clinic attendees (Antenatal and Surgical Outpatient Clinics)

Methodology: A prospective observational study of all the clinic attendees who consented to be included in this study between January and December 2004 was carried out at Aminu Kano Teaching Hospital, Kano, Nigeria. The clinic attendees had pre-test counseling, followed by voluntary testing and post test counseling for those who were sero-positive and negative. The data obtained were analyzed using chisquare test, and analysis for linear trend in proportions was done using chi-square test for linear trend. Odds ratio and $95 \%$ Confidence Interval were determined where appropriate.

Results: The sero-prevalence of HIV infection among the clinic attendees that were screened was 37.5\%. There was higher prevalence of the infection among the low socio-economic class $\left(X_{\text {trend }}^{2}=53.028, P=\right.$ 0.0000). The prevalence of the infection increased with increasing age up to 50 years, and thereafter decreased with increasing age $\left(X_{\text {trend }}^{2}=8.597, P=0.00337\right)$. Ambulatory status $(O R=0.78, C I=0.60-1.01$, $P>0.05)$, and $\operatorname{sex}(O R=1.01, C I=0.80-1.28, P>0.05)$ of the patients did not show statistically significant difference in the prevalence of the infection.

Conclusion: The high prevalence of HIV infection among the clinic attendees that were screened, call for mandatory screening of all antenatal and surgical patients, especially before a procedure is carried out.

Key words: HIV sero-prevalence, surgical specialty outpatients, ELISA,

\section{Introduction}

National sentinel surveillance data from Nigeria showed an increase in the prevalence of HIV infection among clinic attendees from less than $1 \%$ in 1990 to $5.8 \%$ in $2001^{1}$. Similarly, in Kano state the prevalence of the infection was on the increase between 1998 (1121 patients) and in the first 6 months of 2007 (2718 patients) ${ }^{2}$.

In our Hospital, only voluntary testing following pre-test counseling is employed in the management of our patients. This may delay diagnosis of the infection in some of our patients, until they develop fatal or severe opportunistic infections, such as Cryptococcal septicaemia ${ }^{3,4}$.

It has been shown in many studies that HIV infection rate is higher in densely populated and heavily industrialized communities like $\mathrm{Kano}^{4-6}$, and that the risk of transmission of the infection from patients to health workers, and from mother to child is very high among antenatal women and surgical patients if their HIV status is not known, because of the higher probability of breaching the universal precautions ${ }^{4,5}$.

It is against this background that this study was designed, to assess the sero-prevalence of HIV infection among the patients who attended our surgical specialty clinics (Antenatal and Surgical Outpatient Clinics) at Aminu Kano Teaching Hospital, Kano, Nigeria, so as to make recommendations that will influence their future management. 


\section{Methodology}

This prospective observational study was conducted at Aminu Kano Teaching Hospital, Kano, Nigeria, to assess the sero- prevalence of HIV infection among surgical specialty clinic attendees (Antenatal and surgical outpatient clinics) between January and December 2004.

All the clinic attendees during the period of study were counseled by trained counselors, whose efforts made most of the patients (1135patients out of $1162(97.7 \%))$ to consent to voluntary testing. In the laboratory, a Medical Doctor collected whole blood by venepuncture under aseptic condition using the universal precautions. The serum samples of the patients were obtained by centrifuging the whole blood samples, and the collected aliquot (serum) was put in specimen bottles and labeled.

The screening of all the serum samples was carried out using a rapid HIV 1/2 assay (Capillus HIV 1/2). Antibodies reactive samples were confirmed using immunocomb-II HIV 1/2 assay (Orgenic Israel).

The study variables of interest were age, sex, ambulatory and socio-economic status of the patients. The results obtained were recorded using tables. Data analysis was done with EPI-info (CD 380 Atlanta). Categorical data were compared for statistically significant difference using Chi-square test. Analysis for linear trend in proportions was done using Chi-square for linear trend. Odds ratio and confidence intervals were determined where appropriate.

\section{Results}

The sero-prevalence of HIV infection in this review was $37.5 \%$ of the surgical outpatient clinic attendees. The sex of the patients did not show statistically significant difference in the seroprevalence of HIV infection between the male and female patients $(\mathrm{OR}=1.01, \mathrm{CI}=0.80-1.28, \mathrm{P}>$ $0.05)$ Table 1.

The sero-prevalence of HIV infection had direct correlation with increasing age up to the age of 50 years, and thereafter decreased with increasing age $\left(\mathrm{X}_{\text {trend }}^{2}=8.597, \mathrm{P}=0.00337\right)$. Table II.

The sero-prevalence of HIV infection had an inverse correlation with socio-economic status of the patients $\left(\mathrm{X}_{\text {trend }}^{2}=53.028, \mathrm{P}=0.000\right)$. Table III.

The odd of HIV sero-positivity was not statistically significantly different between the ambulant and non ambulant patients $(\mathrm{OR}=0.78, \mathrm{CI}=0.60-1.01, \mathrm{P}$ $>0.05)$. Table IV
Table I: Distribution of HIV sero-positive patients according to sex

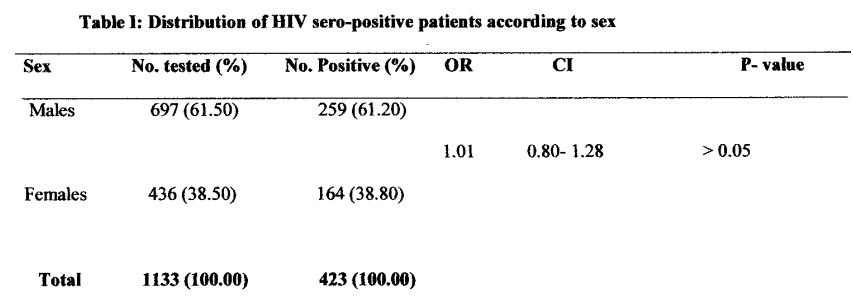

Table II: Distribution of HIV sero-positive patients according to age group

\begin{tabular}{ccr}
\hline Age group & No. Tested (\%) & No. Positive (\%) \\
\hline$<10$ & $163(14.4)$ & $16(3.8)$ \\
$10-19$ & $88(7.8)$ & $37(8.8)$ \\
$20-29$ & $340(30.0)$ & $144(34.0)$ \\
$30-39$ & $339(29.9)$ & $155(36.6)$ \\
$40-49$ & $120(10.6)$ & $52(12.3)$ \\
$>50$ & $83(7.3)$ & $19(4.5)$ \\
Total & $\mathbf{1 1 3 3 ( 1 0 0 . 0 )}$ & $\mathbf{4 2 3}(\mathbf{1 0 0 . 0})$ \\
\hline
\end{tabular}

$\mathrm{X}_{\text {trend }}^{2}=8.597, \mathrm{P}=0.00337$ (Statistically significant)

Table III: Distribution of HIV sero-positive patients according to Socio-economic status

\begin{tabular}{ccc}
\hline Social class & No. Tested (\%) & No. Positive (\%) \\
\hline I & $136(12.0)$ & $12(2.8)$ \\
II & $159(14.0)$ & $46(10.9)$ \\
II & $273(24.1)$ & $82(19.4)$ \\
IV & $318(28.1)$ & $132(31.2)$ \\
V & $247(21.8)$ & $151(35.7)$ \\
Total & $\mathbf{1 1 3 3 ( 1 0 0 )}$ & $\mathbf{4 2 3 ( 1 0 0 )}$ \\
\hline \multicolumn{2}{c}{$\mathrm{X}_{\text {trend }}^{2}=53.028, \mathrm{P}=0.0000$ (statistically significant) }
\end{tabular}

Table IV: Distribution of HIV sero-positive patients according to Ambulatory status

\begin{tabular}{lccccc}
\hline $\begin{array}{l}\text { Status } \\
\text { P-Value }\end{array}$ & No. Tested (\%) & No. & Positive (\%) & OR & CI \\
\hline $\begin{array}{l}\text { Ambulant } \\
>0.05\end{array}$ & $793(70.0)$ & $317(75.0)$ & 0.78 & 0.60 & -1.01 \\
Non-ambulant & $340(30.0)$ & $106(25.0)$ & & & \\
\hline Total & $\mathbf{1 3 3 ( 1 0 0 . 0 )}$ & $\mathbf{4 2 3 ( 1 0 0 . 0 )}$ & & &
\end{tabular}

\section{Discussion}

The sero-prevalence of $37.5 \%$ among the antenatal and surgical outpatient clinic attendees in this review is comparable to $39.4 \%$ from Murtala Mohammed Specialist Hospital, a sister Specialist Hospital also in Kano metropolis ${ }^{2}$, and $37.4 \%$ from Jos, Nigeria ${ }^{3}$. However it is lower than $58.0 \%$ that was reported from Ethiopia ${ }^{4}$, probably because of the influx of tourists from various countries of the world.

An important finding in this study was that $75 \%$ of the patients were healthy looking and ambulant, and could possibly spread the infection in their communities unknowingly if their HIV status is not known. A similar finding was obtained in other studies from Nigeria ${ }^{2,3,5}$, which emphasizes the need to determine the HIV status of all the patients who are seen in the hospitals, especially those that may transfer the infection to health workers and their fetuses, like antenatal and surgical specialty patients. 
The sero-prevalence of HIV infection was found to increase with increasing age up to 50 years, which concurs with the findings of other authors ${ }^{1,4}$. This may probably be because the spread of HIV infection in sub-Saharan Africa including Nigeria is mainly by sexual intercourse ${ }^{3,5,7}$. People in this age group are more sexually active, and are likely to have to have more migrants among them, with its attendant problem of sexual permissiveness and unsafe sex ${ }^{4}$. Also HIV infection assumed a pandemic status in recent times, which could explain the lower incidence among the older age groups.

Some authors ascribe the higher female prevalence in their study, to the wider surface area in the female genital track, while those who found higher male prevalence ascribed it to higher risk of injury to the genital organs in the males during sexual intercourse, which is more during homosexual intercourse ${ }^{4}$, and also to the fact that more drug addicts are found among the males ${ }^{1,3,7}$. This was not the experience in this study, where the odds of having the infection among the male and female patients did not show statistically significant difference, probably because of early marriage, as well as socio-cultural and religious restrictions in our predominantly Islamic community. This calls for close surveillance among both groups of patients.

The higher prevalence of the infection among the low socio-economic class, agrees with the findings of other authors that it is a disease of poverty ${ }^{8,9}$.

\section{Conclusion and Recommendations}

The high risk groups that were found this study were those under 50 years of age, and the low socio-economic class.

The high prevalence of HIV infection among ambulatory surgical specialty clinic attendees, call for a mandatory screening of all our patients, especially before a procedure is carried out, in order to prevent mother to child transmission of the infection, and the spread of the infection of health workers. Also this will offer the patients the opportunity to know their HIV status and take care of their health, as well as benefit from the Highly Active Antiretroviral Therapy which we offer in our hospital.

With post-test counseling, those who are seropositive will be able to control the spread of the infection in their communities, while those who are sero-negative will know how to prevent being infected and remain sero-negative, like practicing safe sex.

\section{References}

1. Federal Ministry of Health. 2001 HIV/Syphilis sentinel sero-prevalence survey in Nigeria. Technical Report April 2002.

2. Ambang L.A. The prevalence of HIV/AIDS in Kano, Kano state, Nigeria. Msc Thesis. 1997.

3. Njoku C.M., Agwale S.M., Dinlinska D.D et al. The rising trend of HIV in patients with AIDS related diseases in Nigeria. A paper presented at national AIDS conference of Brazil. 1997.

4. UNAIDS/AIDSCAP. The status and trends of the Global HIV/AIDS pandemic. Technical report. 1996.

5. Nwokede E.E., Kwaru A.H. The trend of HIV infection in a Nigerian Teaching Hospital, An 18 month evaluation. Bioscience Research Communications. 2000: 1-10.

6. YOSPIS/UNICEF ZONE-D, BAUCHI, NIGERIA. Survey to determine the number and difficulties of HIV/AIDS orphans in Kano state, Nigeria. Technical Report. 2000.

7. Hansen K., Xchapman G., Chitsikel A.G. The costs of HIV/AIDS care at government hospital in Zimbabwe. Health Policy Plan. 2000.

8. Omole-Ohonsi A., Nwokede E.E. HIV and Malaria coinfection in pregnancy. Nigerian Journal of Maternal and Child Health. 2007; 1(1): 6-12.

9. Onah H., Obi S. N., Agbata T.A., Oguanuo T.C. Pregnancy outcome in HIV positive women in Enugu, Nigeria. J. Obstet Gynaecol. 2007; 27(3): 271-274. 